\title{
Leitbild der FMH zur Medizinischen Notfallversorgung in der Schweiz
}

FMH-Forum Notfall*

\section{* Mitglieder \\ FMH-Forum Notfall \\ Prof. em. Dr. med. \\ Wolfgang Ummenhofer, \\ SGAR, Vorsitzender \\ Sonia Barbosa, MHS, \\ Expertin, Departement \\ Dienstleistungen und \\ Berufsentwicklung FMH \\ Dr. Denis Bachmann, SGP \\ Prof. Dr. Serge Bankoul, VBS \\ Dr. Rudolf Hauri, \\ VKS-AMCS \\ Prof. Dr. Simon Jung, SNG \\ Prof. Dr. Dagmar Keller, \\ SGK/SGNOR-Klinische \\ Notfallmedizin \\ Dr. Michel Alexandre \\ Matter, FMH-ZV \\ Dr. Robert McShine, SGPP \\ Dr. Stefan Müller, \\ SGNOR-Präklinische \\ Notfallmedizin \\ Dr. Patrick Siebenpfund, SGI \\ Dr. Paul-Martin Sutter, SGC \\ Dr. Beat Stücheli, SGAIM}

Le direttive della FMH per il settore del salvataggio in Svizzera sono disponibili anche in italiano-sulla pagina Web:

www.fmh.ch $\rightarrow$ Servizi FMH - Forum Urgenza o www.saez.ch $\rightarrow$ Aktuelle Ausgabe $\rightarrow$ Archiv $\rightarrow 2021 \rightarrow 7$.

\section{Präambel}

Das FMH-Forum Notfall ist ein Zusammenschluss der ärztlichen Fachgesellschaften, die sich für eine Optimierung der medizinischen Notfallversorgung in der Schweiz engagieren. Schwerpunkte sind die zukünftige Rolle der ärztlichen Grundversorgerinnen und Grundversorger in der Betreuung ambulanter Notfallpatientinnen und -patienten, die Verbesserung der Rettungskette und der professionelle Rettungsdienst. Aus der Perspektive der FMH werden Modelle und Empfehlungen entwickelt, um die individuelle und professionelle Notfallhilfe, auch bei Grossschadensereignissen, nationalen Bedrohungslagen und Katastrophen, gesamtschweizerisch zu strukturieren sowie einheitlich und rechtlich verbindlich zu regeln. Deshalb sind auch die Vereinigung der Kantonsärzte und Kantonsärztinnen der Schweiz (VKS) und der Koordinierte Sanitätsdienst (KSD) im Forum Notfall vertreten.

Die FMH setzt sich dafür ein, dass die medizinische Notfallversorgung gemeinsam durch speziell weitergebildete Hausärztinnen und -ärzte bzw. Notärzte einerseits und Rettungssanitäterinnen und Rettungssanitäter andererseits gewährleistet wird. Das 1996 von der Plattform Rettungswesen der FMH als Vorläuferin des Forums Notfall publizierte Thesenpapier hat die Notfallversorgung und das Rettungswesen in der Schweiz stark beeinflusst. Das aktualisierte Leitbild soll den Anforderungen an ein zukunftsorientiertes und effizientes Notfalldispositiv für die Schweiz gerecht werden.

\section{Rettungskette}

Der Begriff Rettungskette beschreibt das koordinierte Vorgehen von Ersthelfern, First Respondern und professionellen Rettungsteams.

Den ersten Elementen der Rettungskette, wie dem Erkennen einer Notfallsituation, der Alarmierung, dem Beginn von erster Hilfe und lebensrettenden Sofortmassnahmen, müssen besondere Beachtung beige- messen werden. Dazu ist es unumgänglich, dass die breite Bevölkerung in der Gesundheitskompetenz und im Anwenden von Notfallkompetenzen geschult wird, was auch bedeutet, das Thema «Wiederbelebung» und ausgewählte Kapitel der ersten Hilfe in den obligatorischen Schulunterricht zu integrieren. Über eine Integration in die kantonalen Lehrpläne sollen den Schulen die notwendigen Mittel gesprochen werden. Für die fachlichen Inhalte ist der Swiss Resuscitation Council (SRC) zuständig.

Die vorerst regionalen und vereinzelt kantonalen First Responder-Systeme sollen, wo möglich, überregional weiterentwickelt und mit entsprechender Alarmierung ausgebaut werden. Informationen zur Lokalisierung von Defibrillatoren müssen jedem Ersthelfer unkompliziert über Register zur Verfügung stehen; die Standorte sollen zugänglich, einheitlich beschildert und in die kantonalen Rettungssysteme integriert werden.

\section{Ambulante notfallmedizinische Grundversorgung/Dienstärzte}

Es liegt in der Verantwortung der Kantone, die notfallmedizinische Grundversorgung überall und jederzeit sicherzustellen; ausführendes Organ für diese Aufgabe ausserhalb des Rettungsdienstes sind die kantonalen Ärztegesellschaften. Leistungserbringer sind die niedergelassenen Hausärztinnen und -ärzte, die sich im Bereitschaftsdienst ausserhalb ihrer Praxispräsenz regional als Dienstärzte organisieren. Durch den stetig zunehmenden Mangel an Grundversorger(inne)n in vielen Regionen der Schweiz kann ein flächendeckendes und permanentes Dienstarztnetz bald nicht mehr garantiert werden. Gleichzeitig stellt die Hausarztmedizin einen wichtigen Eckpfeiler in der Grundversorgung einer zunehmend älteren und polymorbiden Bevölkerung dar. Um auch als Zukunftsmodell überlebensfähig zu bleiben, drängt sich für die Funktion der Hausarztmedizin in der ambulanten notfallmedizinischen Grundversorgung eine Reihe von notwendigen Strukturreformen auf. 
Das FMH-Forum Notfall unterstützt die Umsetzung folgender Massnahmen:

- Regionale/überregionale ambulante medizinische Notrufzentralen mit kantonal, besser schweizweit einheitlicher Notfallnummer sind erforderlich.

- Diese Notrufzentralen müssen professionell besetzt sein, den Einsatz von Triagierungs-Algorithmen und die Implementierung ärztlicher Telemedizin sicherstellen.

- Der Betrieb von Ärzte-Netzwerken in Form von Permanencen/Notfallpraxen als zentrale Anlaufstellen für ambulante Notfallpatienten in Agglomerationen muss forciert werden; die räumliche Nähe zu Notfallstationen, d.h. eine enge Vernetzung vom ambulanten zum stationären Bereich, erweist sich auch bei Regionalspitälern als Ressourcenoptimierung.

- Die vermehrte Nutzung interprofessioneller Strukturen, insbesondere die Delegation medizinischer Leistungen an Advanced Practice Nurses (APN) und Physician Associates (PA) (s. 6.6) ist erstrebenswert.

- Ein ärztlicher Hintergrunddienst zur Sicherstellung von Hausbesuchen bei immobilen Patient(inn)en und Heimen muss sichergestellt, aber auch angemessen vergütet werden.

- In Regionen, in denen aus geographischen Gründen ein flächendeckendes Notarztnetz schwierig umzusetzen ist, werden grundversorgende Ärztinnen und Ärzte als Dienstärzte mit entsprechender Ausbildung im Rahmen eines kantonalen Rettungskonzepts eingesetzt (s. 3.2). Dienstärztinnen und Dienstärzte müssen vital bedrohte Patient(inn)en beurteilen und bis zum Eintreffen der Notärztin oder des Notarztes adäquat behandeln können.

\section{Die Bevölkerung soll in der Gesundheits- kompetenz und im Anwenden von Notfall- kompetenzen geschult werden.}

- Häufig müssen Hausärztinnen die Notfallversorgung in psychischen Ausnahmesituationen (z.B. fürsorgerische Unterbringung) übernehmen; hierfür soll eine spezialisierte Weiterbildung der Schweizerischen Gesellschaft für Notfall- und Rettungsmedizin (SGNOR) mit Unterstützung der Foederatio Medicorum Psychiatricorum et Psychotherapeuticorum (FMPP) etabliert werden.

- Die Dienstleistung der notfallmedizinischen Grundversorgung darf für Dienstärztinnen und -ärzte nicht an Attraktivität verlieren. Mittels einheitlicher Reglementierung, entsprechender Weiterbildungsmöglichkeiten und angemessener finanzieller Entschädigung für Ausrüstung, Nacht- und
Pikettdienste soll dem Rechnung getragen werden. Für die finanzielle Ausstattung des regionalen Notfall-Versorgungskonzepts sind die kantonalen Ärztegesellschaften und die Kantone zuständig.

\section{Professioneller Rettungsdienst}

\subsection{Rettungs- und Transportsanitäter(in)}

Die Ausbildung zu Rettungssanitäter(inne)n (RS - Diplom HF) und Transportsanitäter(inne)n (TS - Berufsprüfung, BP) ist durch das Bundesamt für Bildung und Technik (BBT) verbindlich geregelt.

Für einen dringlichen Notfall müssen diplomierte Rettungssanitäter(innen) eingesetzt werden. Deren Grundkompetenz kann durch eine delegierte ärztliche Kompetenz erweitert werden; der Rettungssanitäter und die Rettungssanitäterin tragen hierfür die Durchführungsverantwortung

Auch "einfache» Krankentransporte erfordern eine qualifizierte Betreuung. Die minimale personelle Qualitätsanforderung für geplante Krankentransporte ohne Vitalbedrohung ist die Ausbildung zum Transportsanitäter bzw. zur Transportsanitäterin.

Für die Aus- und Weiterbildungsentwicklung von Rettungs- und Transportsanitäter(inne)n ist das Forum Berufsbildung im Rettungswesen zuständig; dieses trägt auch die fachliche Verantwortung für alle Fragen der Berufsanerkennung.

\subsection{Notärztinnen und -ärzte}

Notärztinnen und Notärzte mit Fähigkeitsausweis Präklinische Notfallmedizin/Notarzt (SGNOR) (FA PKNM) müssen an geeigneten Institutionen jederzeit einsatzbereit, in eine anerkannte Rettungsorganisation eingebunden sein und bei entsprechender medizinischer Indikation auch eingesetzt werden. Diese Indikation kann je nach Einsatzort und -zeit regional modifiziert werden. Das Nicht-Einsetzen der Notärztin oder des Notarztes bei gegebener Indikation muss im jeweiligen Einsatzrapport vermerkt und die Abweichung von den Zertifizierungsgrundlagen (s. 6.4) begründet werden. Bei kantonalen Dienstarztkonzepten sind die Einsatzindikationen verbindlich und müssen entsprechend den Absprachen umgesetzt werden.

Der Notarzt, die Notärztin verfügt über eine spezifische Weiterbildung und eine spezielle Ausrüstung. Für die Weiterbildung zum Fähigkeitsausweis Präklinische Notfallmedizin/Notarzt (SGNOR) ist die SGNOR im Auftrag des Schweizerischen Instituts für Weiter- und Fortbildung SIWF FMH zuständig.

Notarztdienste mit Notärzt(inn)en in Ausbildung (s. 6.5) sollen mit einem repräsentativen prozentualen 
Anteil von erfahrenen Notärzt(inn)en mit abgeschlossenem Fähigkeitsausweis (FA) betrieben werden.

In Agglomerationen sollen grundsätzlich Notarztdienste mit entsprechender Einsatzindikation betrieben werden. Ersatzweise können in geographisch schwierigen Regionen ein Dienstarztkonzept und die Luftrettung den bodengebundenen Rettungsdienst unterstützen.

Schweizweit soll nach einem einheitlichen System ein ambulanter Notarzttarif definiert werden. Dafür ist die SGNOR in Zusammenarbeit mit der FMH und den Tarifpartnern verantwortlich.

\subsection{Sanitätsnotrufzentralen (SNZ)}

Die Sanitätsnotrufzentralen (SNZ) koordinieren überregional die präklinischen Notfalleinsätze. Die SNZ sind untereinander vernetzt und betreiben die Sanitätsnotrufnummer 144 in der ganzen Schweiz flächendeckend und gebührenfrei. Weitere Zusammenschlüsse von regionalen SNZ zu einer überregionalen Rettungszentrale sind wünschenswert, um betriebswirtschaftlich und logistisch eine kritische Grösse zu erreichen.

Informationen zur Lokalisierung von Defibrillatoren müssen jedem Ersthelfer unkompliziert über Register zur Verfügung stehen.

Als Disponenten arbeiten medizinische Fachpersonen mit einer SNZ-spezifischen Weiterbildung. Die medizinische Leitung einer SNZ obliegt einer Notärztin bzw. einem Notarzt SGNOR.

Die SNZ triagieren primär alle Notrufe und bieten die geographisch nächsten adäquaten notfallmedizinischen Primärversorgungsmittel auf.

Je nach Situation

- erhält der Hilfesuchende unterstützende Anweisungen, um die Zeit bis zum Eintreffen der Rettungskräfte optimal zu überbrücken;

- wird die Notfallpatientin oder der Notfallpatient der Dienstärztin bzw. dem Dienstarzt oder einer Notfallpraxis zugewiesen;

- werden der nächste Rettungswagen und, wenn indiziert, die Notärztin, der Notarzt respektive die Dienstärztin, der Dienstarzt alarmiert;

- wird bei gegebener Indikation der Rettungshelikopter eingesetzt;

- ist insbesondere bei pädiatrischen Notfällen primär eine Notärztin oder ein Notarzt und grosszügig die Luftrettung aufzubieten;

- werden bei Bedarf die Einsatzleiter(in) Sanität (EL San) und die Leitende Notärztin bzw. der Leitende Notarzt (LNA) angefordert;
- funktionieren die SNZ bei Grossschadensereignissen und Katastrophen als sanitätsdienstliche Einsatzzentrale (Backoffice).

\section{Klinische Notfallmedizin (KNM) / Notfallstationen (NFS) der Spitäler}

Sämtliche Notfallstationen der Schweizer Spitäler sollen aufgrund der Empfehlungen der SGNOR zu den Minimalanforderungen an Notfallstationen (NFS) [1] einheitlich kategorisiert werden. Um die qualitativ hochstehende Weiterbildung für die Tätigkeit auf den NFS garantieren zu können, müssen Weiterbildungsstätten für den Fähigkeitsausweis Klinische Notfallmedizin (SGNOR) bzw. den interdisziplinären Schwerpunkt Klinische Notfallmedizin (ISP KNM SGNOR) von der SGNOR akkreditiert und in Weiterbildungsstätten Kategorie 1, 2 oder 3 eingeteilt sein. Diese SGNOR-Akkreditierung setzt verschiedene Bedingungen voraus, wie z.B. das Vorhandensein eines Triagesystems, definierte Patientenpfade und Serviceleistungen von anderen mit dem Notfall verbundenen Organisationseinheiten. Weiterhin ist erforderlich, dass (je nach Kategorie) zumindest der oder die Leiter(in) der NFS Träger des ISP KNM SGNOR ist.

Assistenzärztinnen und -ärzte aus den Spitalkliniken rotieren zur Ausbildung auf die Notfallstation oder werden direkt auf der NFS zur Erlangung des SP KNM angestellt.

Die SGNOR-Akkreditierung bedingt, dass je nach Weiterbildungsstätte ein gewisser Prozentsatz der Pflegenden ein Nachdiplomstudium (NDS HF) in Notfallpflege absolviert hat. Interprofessionalität ist eine Voraussetzung für die optimale Patientenbetreuung auf der NFS. Alle NFS weisen ein Katastrophen- und Pandemiekonzept auf, welches regelmässig aktualisiert wird (s. 5).

\section{Grossereignisse und nationale Bedrohungslagen}

Für Grossschadensereignisse und Katastrophen haben der KSD und die SGNOR die nationale Weiterbildungsplattform Sanitätsdienstliche Führung Grossereignis (SFG) etabliert.

Das Schweizerische Zentrum für Rettungs-, Notfall-und Katastrophenmedizin (SZRNK) ist ein neutrales, national und international ausgerichtetes Kompetenzzentrum und organisiert im Auftrag des KSD die SFG-Curricula. Mit der Organisation der französischsprachigen Kurse (CSAM/CEFOCA-6) ist das CHUV beauftragt. Der SFG-PKurs (prehospital) vermittelt die Prinzipien und Grundkonzepte der sanitätsdienstlichen Führung und informiert über Ressourcen und Konzepte der schweizerischen Partnerorganisationen. SFG-H-Kurse (hospital) vermit- 


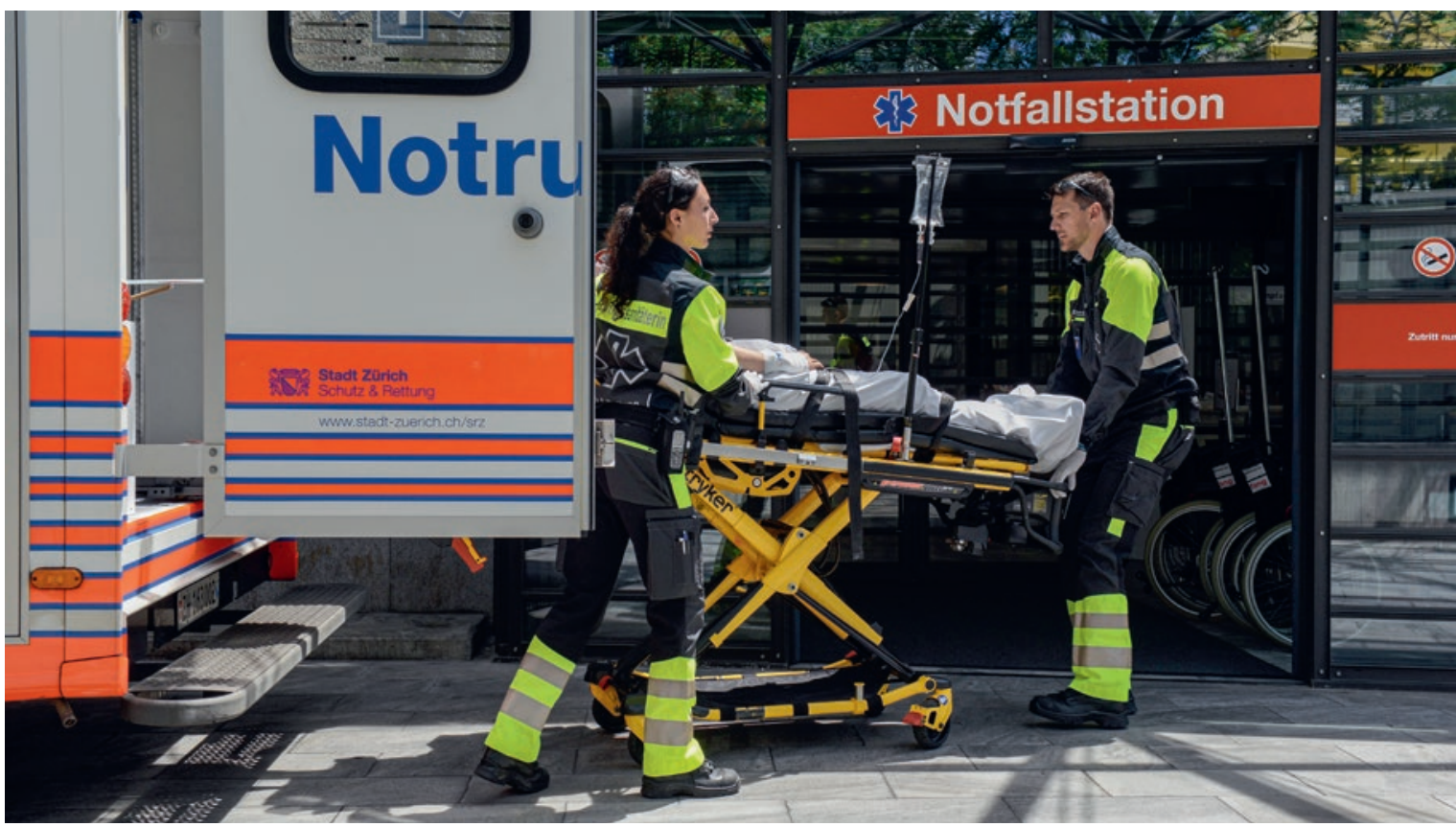

Für einen dringlichen Notfall müssen diplomierte Rettungssanitäterinnen und -sanitäter eingesetzt werden.

teln die Prinzipien der Führung einer NFS bei einem Grossereignis und fokussieren auf die Schnittstelle Präklinik/Klinik.

Rettungssanitäter(innen) und Notärztinnen und -ärzte werden gemeinsam zum bzw. zur «Bereichsleiter(in) Sanität» (BL San) und «Einsatzleiter(in) Sanität» (EL San) resp. «Leitender Notarzt/Leitende Notärztin» (LNA) weiter- und obligatorisch fortgebildet. Die Kantone sind aufgefordert, diese Funktionen einzurichten und mit den notwendigen personellen und materiellen Ressourcen auszustatten.

Im Ereignisfall gibt das internetbasierte Informationssystem (IES) des KSD Informationen über verfügbare Spitalressourcen und unterstützt den Datentransfer zwischen Schadensplatz und Versorgungseinheiten. Damit IES einsatzfähig wird, müssen die beteiligten Dienste und Spitäler instruiert und regelmässig beübt werden.

Im Rahmen der in die Zuständigkeit des Bundes und der Kantone fallenden Pandemiekonzepte muss den grundversorgenden Hausärztinnen und Hausärzten ihre gebührende Rolle zugewiesen werden. Dies setzt die Bereitschaft voraus, sie mit entsprechenden Ressourcen für diese Aufgaben auszustatten. Die Hausärzte sind das wichtigste Bindeglied zu den betroffenen Patient(inn)en, und die übergeordneten Institutionen des Bevölkerungsschutzes müssen sich ihre Erfahrung zunutze machen und die Einbindung der kantonalen Ärztegesellschaften in die kantonalen Krisenorganisationen sicherstellen. In den Pandemiekonzepten muss zusätzlich beantwortet werden, wie die bestehenden Versorgungsstrukturen (niedergelassene Ärztinnen/Spitäler) neben der Versorgung der Pandemiepatienten die medizinische Grundversorgung aufrechterhalten sollen. Die Pandemiekonzepte müssen regelmässig auf ihre Funktionalität überprüft werden.

\section{Qualitätsmanagement/Datenschutz}

\subsection{Hilfsfrist für professionelle Rettungs- strukturen}

Vital bedrohte Notfallpatient(inn)en sollen in der Schweiz innerhalb 15 Minuten von professionellen Rettungskräften erreicht werden (Hilfsfrist); wo immer möglich, ist auf eine Hilfsfrist von 10 Minuten hinzuarbeiten. Bei entsprechender Indikation muss eine Notärztin bzw. ein Notarzt in der gleichen Zeit den Patienten oder die Patienten erreichen können. In geographisch komplexen Regionen kann ein Dienstarztdispositiv das Notarztnetz ergänzen. Das lokale Rettungssystem muss sich mit überregionalen Rettungsstrukturen (Luftrettung) vernetzen.

\section{2 Ärztliche Leitung Rettungsdienst}

Jeder Rettungsdienst verfügt über eine ärztliche Leitung mit Fähigkeitsausweis Präklinische Notfallmedizin/ Notarzt (SGNOR). Die ärztliche Leitung ist in medizinischen Fragen weisungsberechtigt, kann ärztliche 
Kompetenzen an Rettungssanitäter(innen) delegieren und ist für die medizinische Qualitätssicherung des Rettungsdienstes verantwortlich.

\subsection{Zielspital}

Notfallpatient(inn)en sind unter kompetenter Überwachung und Behandlung wenn möglich direkt in das für die definitive Versorgung der schwersten Schädigung geeignete Zielspital einzuweisen. Für spezielle Patientenkollektive (Polytrauma, Schwerbrandverletzte, Stroke, Akutes Koronarsyndrom) sind entsprechende Zentren definiert worden. Jeder Transport ist mit den angemessenen personellen (TS, RS, NA) und technischen Ressourcen (Rettungswagen, Helikopter) durchzuführen. Die ärztliche Leitung eines Rettungsdienstes erstellt ein entsprechendes Zuweisungskonzept für die Spitäler in seinem Einzugsgebiet. Bei langen bodengebundenen Transportzeiten sollen Luftrettungsmittel zugezogen werden.

\subsection{Qualitätskontrolle}

Alle Stufen des Rettungswesens haben einer Qualitätskontrolle zu genügen. Der Interverband für Rettungswesen (IVR) erlässt Richtlinien für Transportmittel und Ausrüstungen der Rettungsdienste. Alle Rettungsdienste in der Schweiz müssen IVR-zertifizert sein oder eine gleichwertige Qualitätsüberprüfung nachweisen. Der vermehrte Aufwand der zertifizierten Rettungsdienste soll sich in der Tarifstruktur der Kostenträger widerspiegeln.

\section{5 Ärztliche Aus-, Weiter- und Fortbildung}

Eine notfallmedizinische Basisausbildung soll an allen Medizinischen Fakultäten der Schweiz mit einem am PROFILES-Katalog der SMIFK orientierten Curriculum einheitlich in das Medizinstudium integriert werden. Die Qualitätskontrolle der notfallmedizinischen Grundkurse hat derzeit das FMH-Forum Notfall übernommen (Dienstärztinnen und -ärzte). Für die ärztliche notfallmedizinische Weiterbildung ist die SGNOR im Auftrag des SIWF zuständig.

Den ISP KNM (SGNOR) und den FA PKNM regelt die SGNOR. Die Absolvierung der für die Weiterbildung zum Notarzt geforderten Notarzteinsätze ist nur an einer akkreditierten Weiterbildungsstätte oder einem anerkannten Notarztstandort möglich.

Für die Weiterbildung zum Leitenden Notarzt / zur Leitenden Notärztin (LNA) resp. Einsatzleiter(in) Sanität (EL San) ist der KSD in Kooperation mit der SGNOR resp. der Vereinigung Rettungssanitäter Schweiz (VRS) zuständig.

Die ärztliche Fortbildung wird vom SIWF zusammen mit der SGNOR bzw. dem KSD geregelt. Das SZRNK soll
Lehre und Forschung sowie Weiter- und Fortbildung in Rettungs-, Notfall- und Katastrophenmedizin stärken.

\subsection{Interprofessionalität}

Die effiziente Vernetzung ärztlicher Strukturen mit interprofessionellen NFM-Ausbildungen (TS, RS, Disponent(inn)en, Notfallpflege/NDS HF) ist ein zentraler Baustein im notfallmedizinischen Spektrum der Schweiz. Für die ambulante Grundversorgung sollten Unterstützungsmodelle mit nicht-ärztlichen medizinischen Fachpersonen (z.B. APN, PA) evaluiert werden.

\subsection{Advanced Care Planning (ACP)}

Damit das Selbstbestimmungsrecht eines Notfallpatienten oder einer Notfallpatientin auch in einer Ausnahmesituation (Bewusstlosigkeit usw.) gewahrt werden kann, sind sogenannte Ärztliche Notfallanordnungen essentiell. Das Forum Notfall setzt sich dafür ein, dass solche Anordnungen mit Unterstützung des Hausarztes bzw. der Hausärztin erstellt und aktualisiert werden, schweizweit möglichst einheitlich formatiert sowie in Notfallsituationen verfügbar und den Angehörigen bekannt sind.

\subsection{Register/Datenschutzbestimmungen}

Sinnvolles Qualitätsmanagement erfordert die Überprüfung etablierter Strukturen anhand relevanter Outcome-Daten. Dies trifft insbesondere für Rettungseinsätze jeglicher Art zu. Diese Daten sind derzeit in der Schweiz aus datenschutzrechtlichen Gründen nicht oder nur sehr eingeschränkt zugänglich. Der oder die Datenschutzbeauftragte des Bundes soll in Zusammenarbeit mit den Kantonen sowie den Fachgesellschaften, beteiligten Institutionen, Spitälern und Rettungsorganisationen Möglichkeiten zur Erfassung von Kenndaten in anonymisierter Form entwickeln. Diese Daten sind auch für den Aufbau und Betrieb von Registern zu spezifischen Notfall-Kollektiven (Polytrauma, Stroke, Herzstillstand/Reanimation) unverzichtbar. Um die aufwendige Erfassung, Verwaltung und Auswertung dieser Datenbanken sicherzustellen, müssen die Finanzierung und das Qualitätsmanagement solcher Register unter Beachtung der gemeinsamen Empfehlungen von $\mathrm{H}+$, ANQ, SAMW, unimedsuisse und FMH [2] explizit geregelt sein.

\section{Bildnachweis}

(c) Schutz \& Rettung Zürich

\section{Literatur}

1 Vorstand der Schweizerischen Gesellschaft für Notfall- und Rettungsmedizin (SGNOR). Empfehlungen zu den Minimalvoraussetzungen einer Notfallstation. Schweiz Ärzteztg. 2014;95(35):1264.

2 www.fmh.ch/files/pdf7/register_empfehlungen.pdf 